\title{
Closed-Form Second-Order Power Transmission Characteristic Model of the Isolated Dual Active Bridge Converter
}

\author{
Yunpeng Yao, Shen Xu, Shengli Lu, and Weifeng Sun
}

\begin{abstract}
The closed-form second order power transmission characteristic (SO-PTC) models of the isolated dual active bridge (IDAB) converter is proposed in all the control strategies in this paper, and the SO-PTC model is derived by the fundamental harmonic approximation (FHA) method. This SO-PTC model shows a better accuracy and a good convenience in predicting the output power of the DAB converter in practical applications. By using this model, the designer can calculate the maximum acceptable parasite resistance of the DAB converter according to the targets of the design; these targets include the efficiency, the voltage conversion ratio, the maximum output power, and so on. Based on the calculated maximum acceptable resistance, the choice of the power switches, the design of PCB and the calculation of the turns on the two sides of the transformer can be facilitated. The validity of the proposed SO-PTC model is verified on a $100 \mathrm{~W}$ prototype.
\end{abstract}

Index Terms-Power transmission characteristic, FHA, model, efficiency, dual active bridge.

\section{INTRODUCTION}

The world-wide increasing demand for the new energy calls for energy storage technique. As an interface between the battery and the power consumption equipment, the bidirectional converters are widely used in power systems like solar electrical energy generation systems, hybrid electrical vehicles, electrical bicycles, wind power generation systems and many other applications which need bidirectional power flows. Among the bidirectional converter, the IDAB converter is attracting more and more attention these years for its properties like zero voltage switching (ZVS), high reliability, low current stress, and good dynamic performance [1]-[3].

To facilitate the design of the IDAB converter, the power transmission characteristics (PTC) of the IDAB converter have been researched in many papers. The PTC model of the traditional single phase shift (SPS) controlled converter has been studied in [3], This PTC model is used widely in the design of the IDAB converter. In order to improve the performance of the SPS control, the dual phase shift control (DPS) is proposed in [4], and the corresponding PTC model is also given by the author who claimed that the maximum output power of the DPS controlled IDAB converter can reach 4/3 times of the SPS controlled IDAB converter. This wrong conclusion in the PTC of the IDAB converter is

Manuscript received April 30, 2015; revised October 19, 2015.

Yunpeng Yao, Shen $\mathrm{Xu}$, Shengli Lu, and Weifeng Sun are with the National ASIC System Engineering Research Center, Southeast University, Nanjing, Jiangsu, 210096, PR China (e-mail: 691054166@qq.com). corrected by the author of [5] who points out that the maximum output power of DPS controlled IDAB is equal to that of the SPS controlled IDAB. The PTC of the extended phase shift (EPS) controlled IDAB and triple phase shift (TPS) controlled IDAB are given in [6]-[8] respectively. The PTCs of almost all the control strategies have been studied. But there are two main shortcomings. At first, all of these PTC models are derived based on the assumption that every piece of the inductor current wave is straight; this assumption makes the model very inaccurate when in high voltage conversion ratio. Secondly, all the PTCs are derived by the direct integration method, which results in a very complex solution especially in the PTC model.

This paper proposes a closed-form second order (SO-PTC) model in all the control strategies which considers the parasite resistance of the IDAB converter. To derive the SO-PTC, the fundamental harmonic approximation method is also proposed. The proposed SO-PTC model is compared with the conventional models to show the overall accuracy of the SO-PTC models.
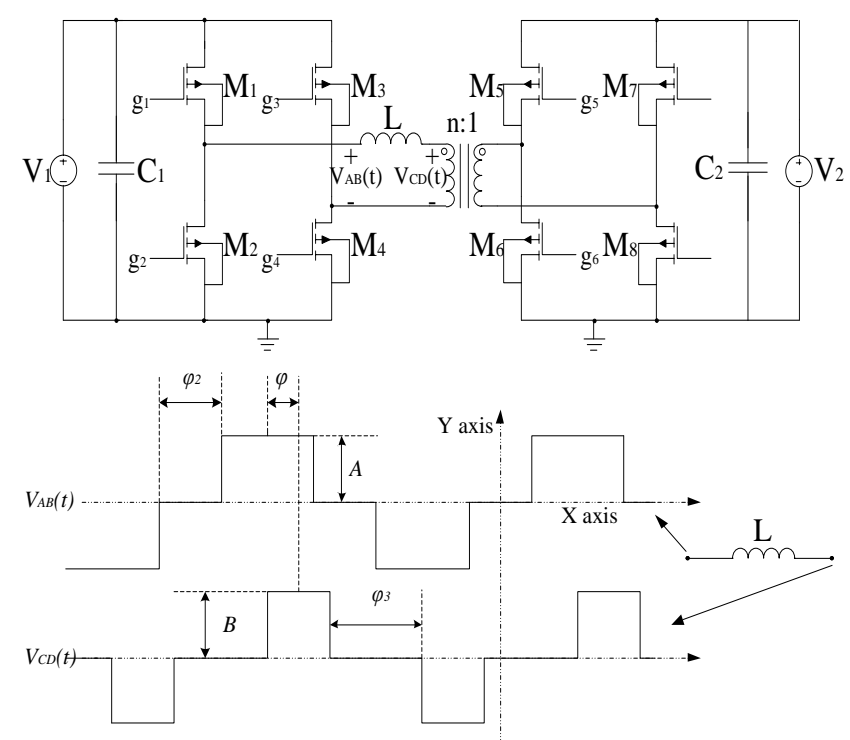

Fig. 1. Schematics; (a) Power stage of an isolated bidirectional IDAB converter; (b) The typical waveform of the IDAB converter.

\section{COnVEntional PTC Models}

The conventional PTC models of IDAB converters include the PTC of the SPS, the PTC of the EPS, the PTC of the DPS, and the PTC of TPS [8]. The PTC of the SPS control is analyzed here to show the limitations of the conventional PTC models for its simplicity. 


\section{A. Conventional PTC Model in SPS Controlled IDAB}

The conventional equivalent circuit of $\mathrm{DAB}$ converter is shown in Fig. 1. In this model, the DAB converter consists of two voltage sources $V_{1}$ and $V_{2}$, two filter capacitors $C_{1}$ and $C_{2}$, two DC/AC bidirectional modules, an inductor $L$ and a transformer. The conventional PTC model of the SPS control is derived by the direct integration method as

$$
P_{\text {in }}=\frac{V_{1} V_{2} n}{L \omega} \varphi(\pi-\varphi) \frac{1}{\pi}
$$

The output power is

$$
P_{\text {out }}=\frac{V_{1} V_{2} n}{L \omega_{s}} \varphi(\pi-\varphi) \frac{1}{\pi}-P_{\text {loss }}
$$

where $\omega_{s}$ is the switching angular frequency, $\varphi$ is the phase shift between $V_{A B}(t)$ and $V_{C D}(t)$ and the $P_{\text {loss }}$ is the total power loss. This model gives the basic ideals of the PTC in IDAB. It can be derived that the output power of the IDAB converter is determined by the input voltage, the output voltage and the phase shift between the $V_{A B}(t)$ and $V_{C D}(t)$ if the power stage has been designed. If these variables are determined, the output power is a constant value no matter how light the output power of the DAB is, and even though the load is zero, there will still be an output power. When the output power is bigger than the consumed power of the load, the output voltage will increase. Finally, the output voltage will be

$$
V_{o}=\frac{V_{1} R_{o} n}{L \omega_{s}} \varphi(\pi-\varphi) \frac{1}{\pi}-P_{\text {loss }} \frac{R_{o}}{V_{o}}
$$

where $R_{o}$ is the resistance of the load. If the load is zero the output voltage will be infinite.

\section{B. Deviations of the Conventional PTC of the SPS Controlled IDAB}

The conventional PTC models will deviate when the output voltage of the IDAB converter increases. The input power calculated by (1) is fixed if parameters of $A, B$, and $\varphi$ are set no matter how bigger the parasite resistance of the IDAB converter will be. This is incorrect because the slope of the inductor current is no longer straight when the parasite resistance of the DAB converter increases. The deviation is shown in Fig. 2.

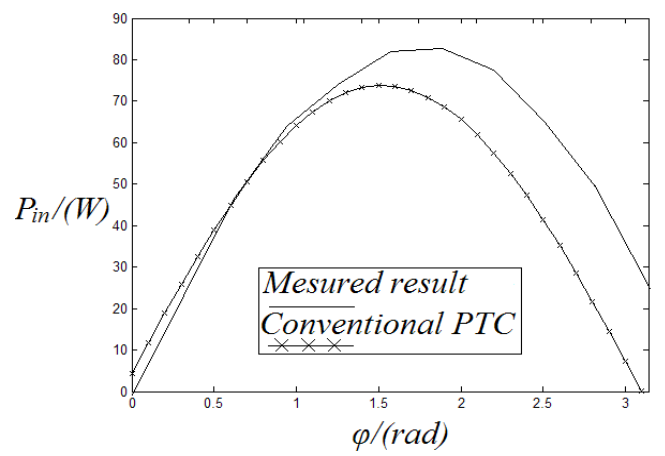

Fig. 2. Deviation of the conventional PTC in predicting the input power of the SPS controlled IDAB converter.

\section{Analysis with the Conventional Model}

An $82 \mathrm{~W}$ IDAB converter is shown in Fig. 3, and its parameters are given in Table I.

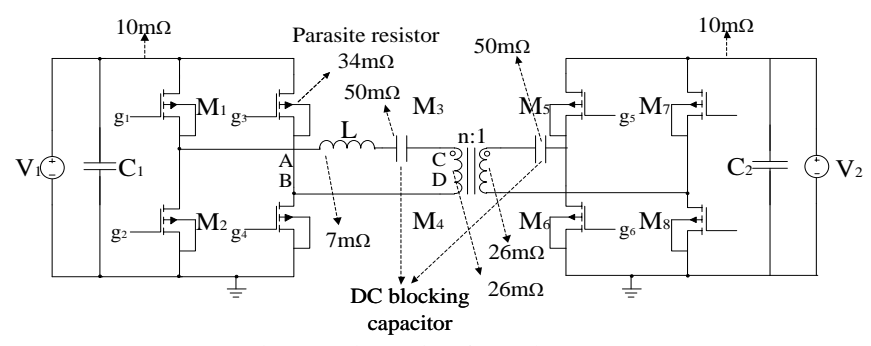

Fig. 3. Schematic of a real system.

TABLE I: MAIN PARAMETERS OF THE REAL SYSTEM IN FIG. 3

\begin{tabular}{|c|c|c|}
\hline$L=L_{\text {leak }}+L_{p}=25.2 \mathrm{uH}$ & $C_{1}=C_{2}=940 \mathrm{uF}$ & $C_{\text {blocking }}=50 \mathrm{uF}$ \\
\hline \multicolumn{3}{|c|}{ Condition: $V_{2}=40 \mathrm{~V} \rightarrow V_{1}=20 \mathrm{~V}$} \\
\hline$f_{s}$ (Switching frequency) $=50 \mathrm{kHz}$ & $n=0.925$ \\
\hline & $R_{\text {loss }}=0.4$ (skin effect is considered) \\
\hline
\end{tabular}

From the measured parameters in Fig. 3 and Table I, the input power can be calculated by (1). The maximum input power is calculated as $75.77 \mathrm{~W}$. The output power is $75.77 \mathrm{~W}-P_{\text {loss. }}$. The total power loss consists of the conduction loss, switching loss, hysteresis core loss, eddy current core loss, auxiliary power supply power consumption and so on. According to [9], the conduction loss can be calculated as

$$
P_{\text {loss }}=R_{\text {loss }} I_{\text {effective }}^{2}
$$

where

$$
R_{\text {loss }}=2 R_{s 1}+R_{L}+R_{t r 1}+R_{\text {block }}+n^{2}\left(2 R_{s 2}+R_{t r 2}+R_{\text {block }}\right)
$$

where $R_{s 1}$ and the $R_{s 2}$ are parasite resistances of the left side MOSFET and right side MOSFET, $R_{t r 1}$ and $R_{t r 2}$ are parasite resistances of the left side winding and right side winding of the transformer, $R_{L}$ is parasite resistance of the inductor, $R_{\text {block }}$ is the parasite resistance of the blocking capacitors, and $n$ is the transformer turn ratio. Because the ZVS is achieved when at the maximum output power and the switching frequency is not high, the ZVS is neglected. Other losses can be classified into the transformer core loss. The core loss can be calculated by the Steinmetz equation [10], which is accurate enough. Then the calculated conduction loss is about $10.5 \mathrm{~W}$, and the core loss is $1 \mathrm{~W}$. So the output power is $64.47 \mathrm{~W}$. The efficiency is about $85 \%$. The calculation result is compared with the test result in Table II. The maximum input power and output power calculated by the conventional PTC model have differences of $6.3 \mathrm{~W}$ with the measured results.

TABLE II: COMPARISONS BETWEEN THE MAXIMUM INPUT AND OUTPUT POWERS OF RESULTS DERIVED FROM THE CONVENTIONAL PTC, AND THE MEASURED RESULTS

\begin{tabular}{|l|l|l|l|}
\hline Calculated & $P_{\text {in }}=75.7 \mathrm{~W}$ & $P_{\text {out }}=64.47 \mathrm{~W}$ & Efficiency $=85 \%$ \\
\hline Measured & $P_{\text {in }}=82 \mathrm{~W}$ & $P_{\text {out }}=69 \mathrm{~W}$ & Efficiency $=84 \%$ \\
\hline
\end{tabular}

\section{IMPROVED MODEL}

\section{A. Fundamental Harmonic Approximation}

The fundamental harmonic approximation has shown its effectiveness in predicting the steady state behavior of the power systems like [11], [12]. The typical DAB converter can 
be modeled as shown in Fig. 1(a), its FHA model is shown in Fig. 4(a). In Fig. 4(a), the DC/AC modules chop the $V_{1}$ and $V_{2}$ into square wave, FHA module extracts the fundamental harmonic of the square wave. Based on the phase difference of the sine waves on the two sides of the inductor, the energies of both $V_{1}$ and $V_{2}$ can be transferred to each other. This approximation is a compromise between accuracy and complexity. In EPS, DPS, and TPS controls, it shows a big convenience in design.

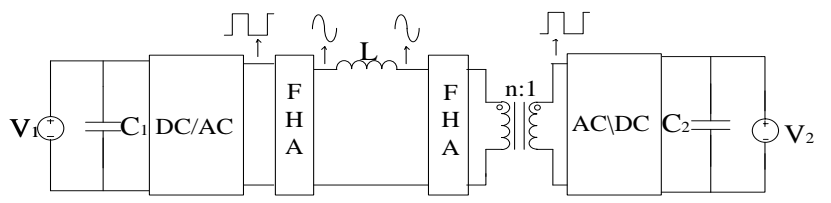

(a)

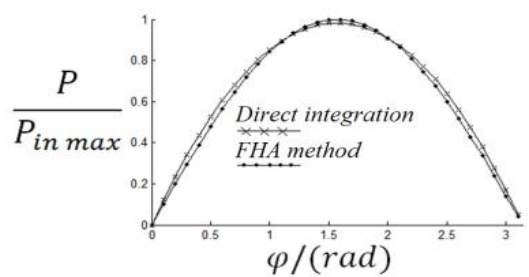

(b)

Fig. 4. FHA model without considering the parasite parameters. (a) The FHA model of the power stage of the IDAB converter. (b) The PTC derived by the FHA model, and it is compared with the PTC derived by the direct integration method.

The transfer function of the FHA module is:

$$
H_{F H A}=\frac{4}{\pi} \cos \left(\frac{\varphi_{2}}{2}\right)
$$

where $\varphi_{2}$ is the second phase shift in the DPS controlled IDAB. The conventional PTC of the SPS controlled IDAB derived from the FHA is shown in Fig. 4(b), and it is compared with the PTC derived by the direct integration method to show its effectiveness.

\section{B. Proposed Model Based on FHA}

The proposed SO-PTC is derived based on the FHA while it considers the total parasite resistance in the derivation as shown in Fig. 5. When using the FHA to calculate the input power, the $V_{A^{\prime} B^{\prime}}(t)$ and $V_{C^{\prime} D^{\prime}}(t)$ can be expressed by $A^{\prime}$ and $B^{\prime} \exp (-j \varphi)$ in the frequency domain, where $A^{\prime}$ and $B^{\prime}$ are amplitudes of $V_{A^{\prime} B^{\prime}},(t)$ and $V_{C^{\prime} D^{\prime}},(t)$. Then the inductor current is

$$
I_{L}(s)=\frac{A^{\prime}-B^{\prime} \cos (\varphi)+j B^{\prime} \sin (\varphi)}{R_{\mathrm{loss}}+j L \omega}
$$

By multiplying (7) with the $A^{\prime}$, and extracting the real part, it is derived that

$$
P_{\text {in }}=\frac{R_{\mathrm{loss}} A^{\prime}\left(A^{\prime}-B^{\prime} \cos (\varphi)\right)+A^{\prime} B^{\prime} L \omega \sin (\varphi)}{2\left(R_{\mathrm{loss}}{ }^{2}+(L \omega)^{2}\right)}
$$

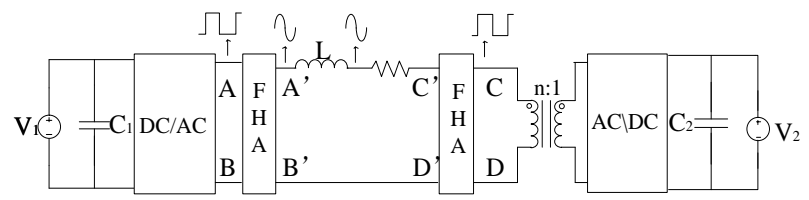

Fig. 5. FHA model of the IDAB converter with consideration of the parasite resistance.
When calculating the output power, $V_{A^{\prime} B^{\prime}}(t)$ and $V_{C^{\prime} D^{\prime}}(t)$ can be expressed by $A^{\prime} \exp (j \varphi)$ and $B^{\prime}$ in the frequency domain. Then the inductor current is expressed as

$$
I_{L}(s)=\frac{A^{\prime} \cos (\varphi)-B^{\prime}+j A^{\prime} \sin (\varphi)}{R_{\mathrm{loss}}+j L \omega}
$$

By multiplying (9) with the $B^{\prime}$, and extracting the real part, it is derived that

$$
P_{\mathrm{out}}=\frac{R_{\mathrm{loss}} B^{\prime}\left(-B^{\prime}+A^{\prime} \cos (\varphi)\right)+A^{\prime} B^{\prime} L \omega \sin (\varphi)}{2\left(R_{\mathrm{loss}}{ }^{2}+(L \omega)^{2}\right)}
$$

The conduction loss can be calculated by subtracting (10) from (8), so the conduction loss is

$$
P_{\text {conduction loss }}=\frac{R_{\mathrm{loss}} A^{\prime 2}+R B^{\prime 2}-2 R_{\mathrm{loss}} A^{\prime} B^{\prime} \cos (\varphi)}{2\left({R_{\mathrm{loss}}}^{2}+(L \omega)^{2}\right)}
$$

In the TPS control, the $A^{\prime}$ and $B^{\prime}$ can be expressed by

$$
\begin{aligned}
& A^{\prime}=A \frac{4}{\pi} \cos \left(\frac{\varphi_{2}}{2}\right) \\
& B^{\prime}=B \frac{4}{\pi} \cos \left(\frac{\varphi_{3}}{2}\right)
\end{aligned}
$$

The improved PTCs of SPS, EPS, DPS controls can be derived by varying the $\varphi_{2}$ and $\varphi_{3}$. From (7), it can be found that the input power is not only determined by the input and output voltages but also determined by the total parasite resistance of the IDAB. This is why the conventional PTC model fails to predict the actual input power of the IDAB. The conduction loss of (11) is not significantly affected by the parasite resistance. Because in most designs, $(L \omega)^{2}$ is usually much larger than $R_{\text {loss }}{ }^{2}$. The typical input power and output power of SPS controlled IDAB are shown in Fig. 6. Notice that, the maximum output power is not exactly at $\pi / 2$ because the effect of the parasite resistance. The difference between the input power and the output power is the conduction loss. In real application, the core loss of the transformer should be considered. The core loss should be subtracted from the calculated output power to derive the real output power.

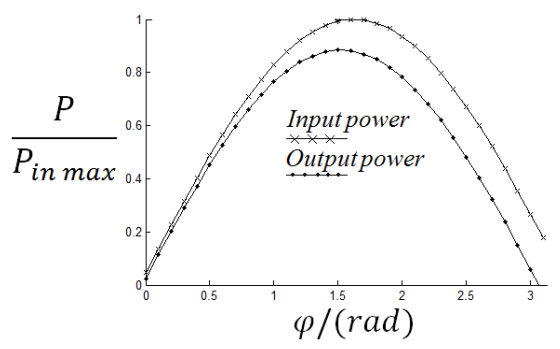

Fig. 6. A typical SO-PTC of the IDAB converter.

\section{EXPERIMENTAL VERIFICATION}

In order to verify the validity of the SO-PTC model, a prototype of the power-stage of a $100 \mathrm{~W}$ DAB converter was 
built. The power-stage was built by using IRFP4232 MOSFETs, IR2113, 470uF-electrolytic capacitors, 50uF-CBB capacitors, toroidal inductor, PC40EE42 transformer (TDK). The schematic of the test system is shown in Fig. 7. This prototype is tested through hand adjustment. The double delay module controls the phase shift of $\varphi_{2}$ while the $\varphi$ can be controlled by the wave generator. The parameters of the prototype are shown in Table III. The parasite resistance distribution is shown in Fig. 3, and it is measured by a micro ohm meter with accuracy of $0.1 \mathrm{~m} \Omega$. The inductances of the inductor and the leakage inductance are all measured with accuracy of $0.1 \mathrm{uH}$.

The test results in Fig. 8 show that the accuracy of the SO-PTC model in predicting the input power in condition 1 is much better than conventional PTC model (difference of $1.2 \%$ VS difference of $8.1 \%$ at $\varphi=\pi / 2$ ). The comparisons between the maximum input and output powers of the measured results, the results of conventional PTC, and the results of proposed SO-PTC are shown in Table IV. When using the conventional analysis based on (1) and (2), the output powers in condition 1 and condition 2 are same no matter how bigger the total parasite resistance of the IDAB will be; it is absolutely different when using the SO-PTC model. When using the SO-PTC model, the difference between the output powers in condition 1 and condition 2 was reflected. So the SO-PTC model is very effective in predicting the PTC of the IDAB converter whose parasite resistor can't be neglected.

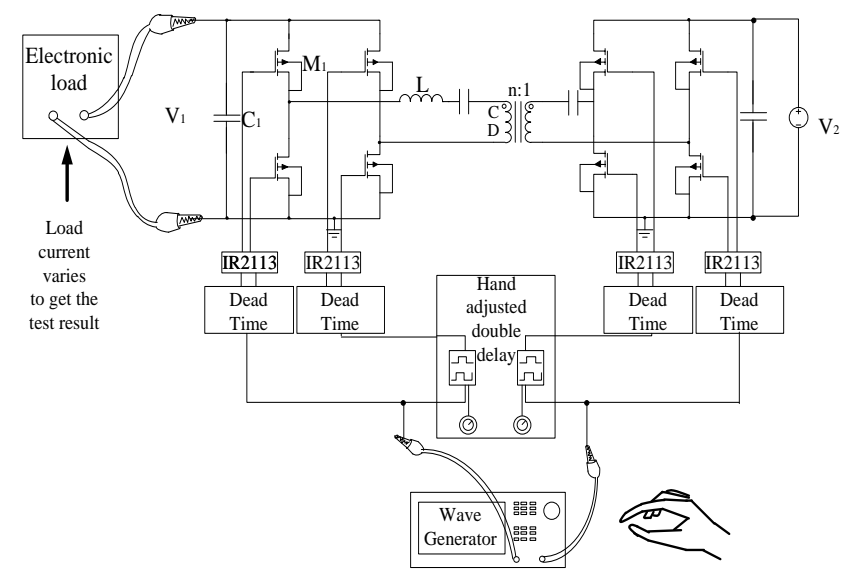

Fig. 7. Schematic of the test system.

TABLE III: MAIN PARAMETERS OF THE PROTOTYPE

\begin{tabular}{|c|c|c|}
\hline$L=L_{\text {leak }}+L_{p}=25.2 \mathrm{uH}$ & $C_{1}=C_{2}=940 \mathrm{uF}$ & $C_{\text {blocking }}=50 \mathrm{uF}$ \\
\hline \multicolumn{3}{|c|}{ Condition $1: V_{2}=40 \mathrm{~V} \rightarrow V_{1}=20 \mathrm{~V}$} \\
\hline \multicolumn{3}{|c|}{ Condition $2: V_{2}=20 \mathrm{~V} \rightarrow V_{1}=40 \mathrm{~V}$} \\
\hline$f_{s}$ (Switching frequency) $=50 \mathrm{kHz}$ & $n=025$ \\
\hline \multicolumn{2}{|c|}{$R_{\text {loss }}=0.4 \Omega$ (skin effect is considered) } \\
\hline
\end{tabular}

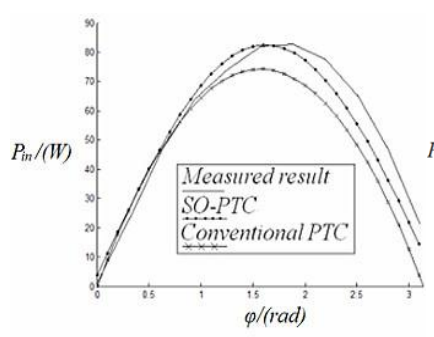

(a)

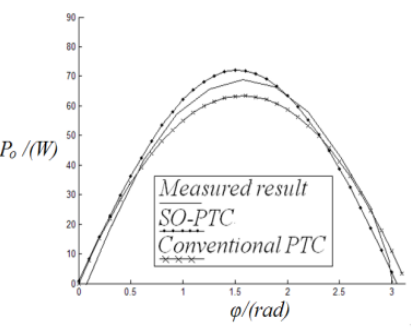

(b)

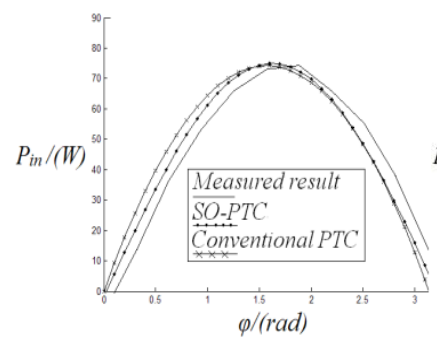

(c)

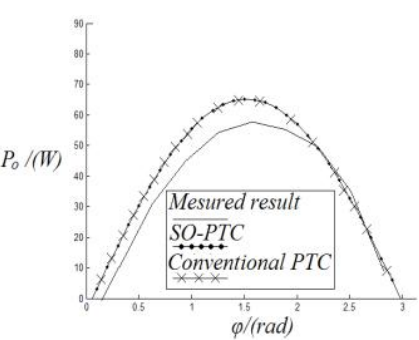

(d)
Fig. 8. Comparisons between the measured results, the results of the proposed SO-PTC, and the results of the conventional PTC. (a). Input power in condition 1; (b). Output power in condition 1; (c). Input power in condition 2; (d). Output power in condition 2.

TABLE IV: COMPARISONS BETWEEN THE MAXIMUM INPUT AND OUTPUT POWERS OF THE RESULTS OF THE CONVENTIONAL PTC, THE RESULTS OF THE PROPOSED SO-PTC, AND THE MEASURED RESULTS

\begin{tabular}{|l|l|l|l|}
\hline \multicolumn{5}{|l|}{$\begin{array}{l}\text { Condition 1 } \\
\text { Conventional }\end{array}$} & $\begin{array}{l}P_{\text {in } \max } \\
=75.7 \mathrm{~W}\end{array}$ & $\begin{array}{l}P_{\text {out } \max } \\
=64.47 \mathrm{~W}\end{array}$ & $\begin{array}{l}\text { Efficiency } \\
=85 \%\end{array}$ \\
\hline SO-PTC & $\begin{array}{l}P_{\text {in } \max } \\
=82.8 \mathrm{~W}\end{array}$ & $\begin{array}{l}P_{\text {out } \max } \\
=72 \mathrm{~W}\end{array}$ & $\begin{array}{l}\text { Efficiency } \\
=85 \%\end{array}$ \\
\hline $\begin{array}{l}\text { Measured } \\
\text { Results }\end{array}$ & $\begin{array}{l}P_{\text {in } \max } \\
=82 \mathrm{~W}\end{array}$ & $\begin{array}{l}P_{\text {out } \max } \\
=69 \mathrm{~W}\end{array}$ & $\begin{array}{l}\text { Efficiency } \\
=84 \%\end{array}$ \\
\hline Condition 2 & \multicolumn{5}{|l|}{} \\
\hline $\begin{array}{l}\text { Conventional } \\
\text { PTC }\end{array}$ & $\begin{array}{l}P_{\text {in } \max } \\
=75.7 \mathrm{~W}\end{array}$ & $\begin{array}{l}P_{\text {out } \max } \\
=64.4 \mathrm{~W}\end{array}$ & $\begin{array}{l}\text { Efficiency } \\
=85 \%\end{array}$ \\
\hline SO-PTC & $\begin{array}{l}P_{\text {in } \max } \\
P_{\text {out } \max }\end{array}$ & $\begin{array}{l}\text { Efficiency } \\
=87 \%\end{array}$ \\
\hline Measured & $\begin{array}{l}P_{\text {in } \max } \\
=74.5\end{array}$ & $\begin{array}{l}P_{\text {out } \max } \\
=59 \mathrm{~W}\end{array}$ & $\begin{array}{l}\text { Efficiency } \\
=79.7 \%\end{array}$ \\
Results & &
\end{tabular}

\section{REFERENCES}

[1] R. W. DeDoncker, M. H. Kheraluwala, and D. M. Divan, "Power conversion apparatus for DC/DC conversion using dual active bridges," U.S. Patent 5027264, Jun. 25, 1991.

[2] C. Mi, H. Bai, C. Wang, and S. Gargies, "Operation, design and control of dual H-bridge-based isolated bidirectional dc-dc converter," IET Power Electron., vol. 1, no. 4, pp. 507-517, Apr. 2008.

[3] M. N. Kheraluwala, R. W. Gascoigne, D. M. Divan, and E. D. Baumann, "Performance characterization of a high-power dual active bridge DC-to-DC converter," IEEE Trans. Ind. Appl., vol. 28, no. 6, pp. 1294-1301, Nov./Dec. 1992.

[4] H. Bai and C. Mi, "Eliminate reactive power and increase system efficiency of isolated bidirectional dual-active-bridge DC-DC converters using novel dual-phase-shift control," IEEE Trans. Power Electron., vol. 23, no. 6, pp. 2905-2914, Nov. 2008.

[5] B. Zhao, Q. Song, and W. Liu, "Power characterization of isolated bidirectional dual active bridge dc-dc converter with dual-phase-shift control," IEEE Trans. Power Electron., vol. 27, no. 9, pp. 4172-4176, Sep. 2012.

[6] B. Zhao, Q. Yu, and W. Sun, "Extended-phase-shift control of isolated bidirectional dc-dc converter for power distribution in micro-grid," IEEE Trans. Power Electron., vol. 27, no. 11, pp. 4667-4680, Nov. 2012.

[7] F. Krismer and J. W. Kolar, "Closed form solution for minimum conduction loss modulation of DAB converters," IEEE Trans. Power Electron., vol. 27, no. 1, pp. 174-188, Jan. 2012.

[8] B. Zhao et al., "Overview of dual-active-bridge isolated bidirectional DC converter for high-frequency-link power-conversion system," IEEE Transactions on Power Electronics, vol. 29, no. 8, pp. 4091-4106, Aug 2014.

[9] F. Krismer and J. W. Kolar, "Accurate power loss model derivation of a high-current dual active bridge converter for an automotive application," IEEE Trans. Ind. Electron., vol. 57, no. 3, pp. 881-891, 2010.

[10] J. Reinert, A. Brockmeyer, and R. W. De Doncker, "Calculation of losses in ferro- and ferrimagnetic materials based on the modified Steinmetz equation," IEEE Trans. Ind. Appl., vol. 37, no. 4, pp. 1055-1061, Jul./Aug. 2001.

[11] S. T. Microelectronics, "LLC resonant half-bridge converter design guideline," Application Note AN-2450, pp. 1-7, 2007. 
[12] G. C. Hsieh, C. Y. Tsai, and S. H. Hsieh, "Design considerations for LLC series resonant converter in two-resonant regions," in Proc. IEEE PSEC'07, 2007, pp. 731-736.

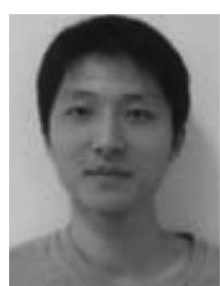

Yunpeng Yao received the B.S. degree from Chengxian College, Southeast University, Nanjing, China, 2010. He is currently working toward the Ph.D. degree at the National ASIC System Engineering Research Center, Southeast University. His current research interests include analog integrated circuits design and multimode control of the power electronic system.

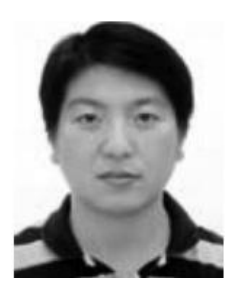

Shen $\mathrm{Xu}$ received the B.S. and Ph.D. degrees in electronics engineering from Southeast University, Nanjing, China, in 2002 and 2011, respectively. He joined the School of Electronic Science and Engineering, Southeast University, in 2011, where he is currently a lecturer. His research interests include nonlinear modeling of power converters, simulations, and power integration.

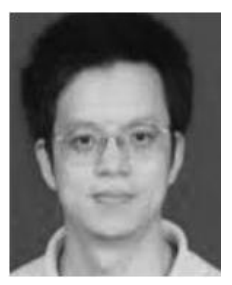

Shengli $\mathbf{L u}$ received the $\mathrm{Ph} . \mathrm{D}$. degree in information and physics from Nanjing University, Nanjing, China, in 1994. Since 1994, he has been with the School of Electronic Science and Engineering, Southeast University, Nanjing, China, where he is currently a professor of the National ASIC System Engineering Research Center. His research interests include VLSI and application specific integrated circuit.

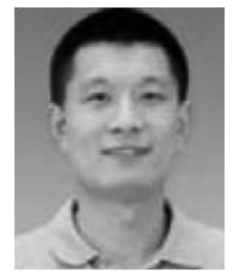

Weifeng Sun received the B.S., M.S., and Ph.D. degrees in electronic engineering from Southeast University, Nanjing, China, in 2000, 2003, and 2007, respectively. Since 2006, he has been with the School of Electronic Science and Engineering, Southeast University, where he is currently a professor and the vice-dean of the School of Electronic Science and Engineering. His research interests include new power device design, power IC and RF device design, power device model, and power system. 